\title{
The importance of being first: A tachistoscopic study of the contribution of each letter to the recognition of four-letter words*
}

\author{
BARBARA A. ERIKSEN and CHARLES W. ERIKSEN \\ Unirersity of Ilinois, Urbana-Champaign, Illinois 61820
}

\begin{abstract}
Three studies investigated the effect on the response time for voicing a four-letter word of delaying one of the letters or the entire word for intervals of up to $500 \mathrm{msec}$. Experiment I found delay of the first letter most detrimental, while delay of the second, third, or fourth letter facilitated performance. Experiment II confirmed these findings and indicated that delay of the entire word produced response times similar to delay of the first letter. Experiment III investigated the possibility that knowledge of the pronunciation of the first letter was the essential factor in facilitating performance when later letters were delayed. It was concluded that when pronunciation of the first letter was known, Ss were able to begin processing the word immediately.
\end{abstract}

The purpose of the present experiments was to analyze the processes involved in the recognition of a single word by skilled readers. It is generally acknowledged that a skilled adult reader uses "higher order units" than words (Gibson, 1967) in reading the printed page. But it is also a rather obvious fact that the child, in learning to read, must at first proceed one word at a time. Although linguists continue to argue about whether the "word" is the "natural perceptual unit of spoken language [Jones, 1965, p. 44]," it is apparent that our graphic representations are organized around words.

Since the studies of Cattell in the latter part of the 19 th century (Huey, 1908), it has been apparent that words are perceived somehow differently from single letters. Cattell reported that whole words could be recognized as quickly as single letters and that the response to a word could occur at a shorter latency than the response to a single letter. Cattell concluded that reading was done by whole-word units. This same conclusion has been reached by Reicher (1969) and Wheeler (1970). The question arises as to what cues are available in words which enable Ss to make these rapid judgments. Studies have concentrated on two lines of inquiry: cues related to letters as visual cues and cues related to sound-spelling correspondences.

\section{Visual Cues}

The shape of the word, which depends on the pattern of letters, was proposed as the main means of recognition by Erdman and Dodge (Huey, 1908). Goldsheider and Müller (Huey, 1908) found that certain "determining" letters seemed to be more important to

*This research was supported by Public Health Service Research Grant MH-1206 and United States Public Health Service Research Career Program Award K6-MH-22014 to the second author. The paper is based on a thesis submitted by the first author to the University of Illinois in partial fulfillment of the requirements for her master's degree. word recognition than others. By presenting word skeletons for identification, they found that the first letter was nearly always necessary for recognition and that other determining letters were those which supplied clues to the word sound.

Using words with deliberate transpositional spelling errors, Bruner and O'Dowd (1958) found that errors at the beginning of words were most disruptive (producing longer identification latencies), errors at the end were next, and errors in the middle of the words were least bothersome. These effects were attributed to differences in "informativeness" of the word parts. Broerse and Zwaan (1966) wondered whether the "first-letter" effect was attributable to the sequential order of speech or to the amount of information present in the first letters as proposed by Sumby and Pollack (1954). They controlled for the frequency of beginning and ending $n$-grams in their language (Dutch) and for the number of words in which an n-gram appeared. Ss were given either the first or last two letters of seven-letter nouns to "solve" in $1 \mathrm{~min}$. Even with information held constant, the first letters were found to lead to quicker solutions. Ss were also able to enumerate more words given beginning n-grams, although more words were possible using an ending $11-$ gram.

In a study in which sequential pronunciation was not a factor, Oléron (1970) used an artificial alphabet to determine whether letters with "tails" (such as b, p, etc.) would contribute more to recognition of "words" when placed in certain positions. He, too, found the first position most important, the last position next, and the middle least important. Melville (1957) also found the first letter most important.

\section{Sound-Spelling Correspondence}

Although the visual pattern of the word is undoubtedly important, many studies in which spelling-sound correspondences have been utilized have suggested that Goldsheider and Müller were on the right 
track. Bishop (1964) found that learning letter-sound correspondences facilitated word reading in a new language (Arabic) more than did the whole-word learning approach. Pronounceable pseudowords are significantly easier to identify (Gibson, Pick, Osser, \& Hammond, 1962), and even first, second, and third graders can identify pronounceable trigrams better than unpronounceable ones (Gibson, Osser, \& Pick, 1963). The pronounceable words, of course, conform to English rules of spelling. Is this effect due only to the spelling rules or are the sounds important? Gibson, Shurcliff, and Yonas (1966) used the pronounceable and unpronounceable pseudowords on deaf college students. The results still showed better performance for pronounceable pseudowords, although not as markedly as for hearing Ss. This may indicate separate contributions from spelling patterns independent of sound correspondences. However, since the students could speak, it is not entirely clear that some form of covert speech-like activity was not occurring.

It has been frequently pointed out that spelling-to-sound correspondence does not mean letter-sound correspondence. Weir and Venezky (in Goodman, 1968) studied English orthography in the hopes of finding relationships to sound that would be useful in the teaching of reading. They concluded that "simple grapheme-to-phoneme correspondences are not only unproductive for the prediction of sound from spelling but also are in conflict with the underlying system of the orthography [p. 198]." Within words, it is the combinations of letters according to the rules of spelling that govern the particular sounds that the letters will have. Black (1954) analyzed phonemic translations of one- and two-syllable words to discover the amount of information transmitted in bits. He found that the average information per sound was higher for shorter words and higher also when going from left to right. Transitional left-right probabilities in English reduce the probability to about 1 in 10 that a particular sound will occur next and to as little as 1 in 3 for some sequences. These results may explain the first-letter dominance effect as well as the failure of Broerse and Zwaan to find an "information" effect when the information controlled was not specifically related to sound.

The question still remains as to whether the reader first perceives the word as a visual unit and then analyzes the sounds or whether he may be able to use his knowledge of spelling-sound patterns to move along by some other method. Aderman and Smith (1971) argue that pronounceability would not confer unity on a word because pronunciation must follow identification rather than the reverse. This does not mean, however, that recognition that the first letters of a word were "impossible" by the rules of orthography could not destroy the classification of a group of letters as a word. This would require the hypothesizing of some kind of sequential left-right processing. Serial processing of one letter at a time from left to right has been rejected on the grounds that complete processing of a single letter appears to take about $200-300 \mathrm{msec}$, a rate obviously too slow for reading (Kolers \& Katzman, 1966). Common sense also tells us that we do not read letter names and then translate them into words and sounds.

The evidence for first-letter importance and the sequential dependencies in sound-spelling correspondence do suggest some kind of left-right processing, however, although not necessarily on a letter-by-letter basis. But does this kind of sequential sound-reading occur only after the word has been recognized as a unit? If so, delaying presentation of one of the letters of a word should retard an S's response to the word relative to his speed of response when the entire word was present. In particular, delay of the first or last letter, both of which have been previously shown to be more important to word identification than the middle letters, should retard responses. The first experiment reported utilized four-letter words, any letter of which could be changed to produce a different English word, to investigate those possibilities.

\section{EXPERIMENT I}

\section{Method}

\section{Subjects}

Ss were three college-age students (one female) and one male high school student, all of whom had normal or corrected to normal vision. They were paid to participate, and all had had previous experience as $\mathrm{Ss}$ in visual experiments.

\section{Apparatus and Stimuli}

Stimuli were presented using a Scientific Prototype Model GA three-field tachistoscope in which the original lamps had been replaced with Sylvania F4T5/CWX bulbs. All three fields were maintained at a luminance of $6 \mathrm{~mL}$ throughout the experiment.

Initiation of the stimulus by $\mathbf{S}$ started a Hunter Klockounter. A microphone located below the S's viewing hood triggered a Scientific Prototype $761-G$ voice relay, stopping the Klockounter. Voice reaction time (RT) was recorded in milliseconds.

Stimuli were constructed from 10 four-letter base words, each of which could be changed by varying any one of the four letters to form a new word. These 10 words and their four sets of variants are presented in Table 1.

Letters for the stimulus words were capitals obtained from Para-tipe pressure letters, 18-pt Futura Bold style. These were placed on transparent vinyl cards. This permitted the use of Fields 2 and 3 of the tachistoscope for display of the words and the delayed letter. Since these fields reverse the displayed stimulus, inserting of the transparent card backwards into the holders produced a correctly oriented image. A white vinyl card was inserted in each holder to provide contrast for the lettering.

For those blocks of trials in which all letters appeared simultaneously, all letters were placed on a single card and appeared in Field 2. When a letter was to be delayed, the three initial letters were placed on the card shown in Field 2 and the single delayed letter was placed on a separate card and displayed in Field 3.

The stimulus field was $51 / 2$ in. wide and $3-9 / 16$ in. high. At the viewing distance of 48 in.. the field subtended visual angles of 6.876 deg $x 4.45$ deg. Fixation was provided by two small dots. 
$1.13 \mathrm{deg}$ apart horizontally, centered both vertically and horizontally in the stimulus field. The words appeared centered between these dots and subtended $.7 \mathrm{deg}$ of visual angle.

Viewing was monocular with the right eye (all Ss were righteye dominant). This made it easier to adjust the delayed letter so that it appeared correctly spaced in relation to the other three letters of the word.

\section{Procedure}

The words were presented with all letters appearing simultaneously or with one letter delayed by $10,20,40,60,80$, 100,150 , or $300 \mathrm{msec}$, yielding nine different presentation conditions. The tachistoscope timers were wired so that the fixation field remained on until the delayed letter appeared. This field then went off until both of the stimulus fields had gone off together. Having two fields on at all times during the stimulus presentations insured equal luminance. In the case of simultaneous presentation of all letters on one card, the fixation field went off, and the blank third field came on as soon as the word appeared in Field 2. Total duration of the stimulus presentation was $2 \mathrm{sec}$. Onset of the stimulus was initiated by $S$ using a hand trigger, following a ready signal from $E$.

Ss were instructed to fixate the area between the two dots and press the hand switch when they felt ready. They were told to say the word that appeared loudly and clearly as soon as they were sure what it was. Each $S$ took part in two practice sessions in order to become familiar with the procedure and with all of the stimulus words. A brief warm-up, using the simultaneous condition, preceded each experimental session. Accuracy was stressed. If an error occurred, the trial was rerun later. Few errors occurred, since the Ss had learned during practice to inhibit the chief source of errors-guessing on some of the longer delay intervals.

During each experimental session, the simultaneous presentation and eight delay times were presented in blocks of 10 trials (90 trials per session). Within each block, the same condition was used and the same letter was delayed (first, second, third, or fourth). One-half of the trials used the base word and one-half used the variants for the appropriate letter. Across experimental sessions, each base word and its four variants appeared equally often under each condition. Twenty-seven different random orders were constructed for order of appearance of words within blocks, all of which were used for an $\mathrm{S}$ before any was repeated. When repeated, they were not used with the same letter delay by condition combinations, so that the possibility of an S's learning a particular order was nil. Order of presentation of conditions by letter-delay combinations within and across sessions was randomized for each S. Sixteen experimental sessions were run, yielding 40 trials per set of variants per ISI per delay condition for each S, or 360 trials per base/variant combinations.

Table 1

Base Words and Variants Used in Experiment I

\begin{tabular}{|c|c|c|c|c|}
\hline \multirow{2}{*}{$\begin{array}{l}\text { Base } \\
\text { Words }\end{array}$} & \multicolumn{4}{|c|}{ Letter Delayed } \\
\hline & 1 & 2 & 3 & 4 \\
\hline LANE & PANE & LoNE & LAKE & LAND \\
\hline FARE & DARE & FIRE & FADE & FARM \\
\hline RICE & $\underline{N I C E}$ & RACE & RIDE & $\mathrm{RICH}$ \\
\hline HARD & $\underline{\text { YARD }}$ & HERD & HAND & HARP \\
\hline BIND & $\underline{\text { FIND }}$ & BAND & BIRDD & BINS \\
\hline LURE & CURE & LQRE & LUTE & LURK \\
\hline BEAT & $\underline{\text { HEAT }}$ & BOAAT & BENT & BEA $\underline{R}$ \\
\hline LAME & FAME & LIME & LACEE & LAMB \\
\hline MATE & $\underline{\text { SATE }}$ & MUTE & MAREE & MATS \\
\hline SLOW & BLOW & STIOW & SLEWW & SLOT \\
\hline
\end{tabular}

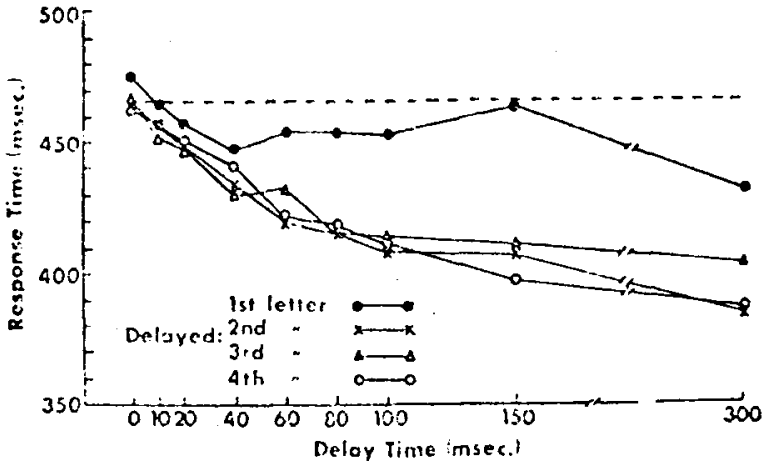

Fig. 1. Mean response time as a function of letter delayed and delay interval. (Delay time subtracted from response time.)

\section{Results and Discussion}

Response times from the onset of the delayed letter for the four letter-delay conditions, averaged across Ss, are presented in Fig. 1. All responses for the base word SLOW and its variants were discarded for Fig. 1 and the analysis which follows, since one $S$ had an apparent malocclusion which prevented a firm enough response to words beginning with SL to stop the timer.

As can be seen from Fig. 1, delaying the first letter produced longer RTs than delaying any of the other three letters. A three-way ANOVA (SS by Letter Delayed by Delay Interval) showed the effect of letter delay to be significant $[F(3,9)=22.65, p<.001]$. The other main effects were also significant. No interactions reached significance. A further three-way ANOVA, using only data for the delay of the second, third, and fourth letters, showed these three delay conditions to be not significantly different $[F(2,6)=.7571, p>.50$. Thus, the significant effect is due mainly to the difference in RT when the first letter is delayed.

Further examination of Fig. 1 reveals that delay of the second, third, and fourth letters does not increase RT to the total word by an amount equal to the delay time, nor does RT to delay of the first letter quite reach that time. Subtracting the delay interval from the response time would give approximately the dashed line in Fig. 1 if this were the case.

Previous work in RT in our laboratory had indicated a possible "priming" effect by a first stimulus upon response to a second stimulus. It was thought that perhaps the fact that none of the response times was longer than the time recorded for the whole word presentation plus the delay interval was due to a "priming" effect on the visual or response system by the appearance of the first three letters. Before attempting to interpret the results, a second experiment was run to check for such an effect.

If a "priming" effect could be obtained, it should be possible to speed response times to the whole word by such a procedure. In Experiment II, two conditions utilized delay of the entire word. In one case, a bar marker appeared immediately upon the S's pressing the switch. The word appeared either simultaneously or 


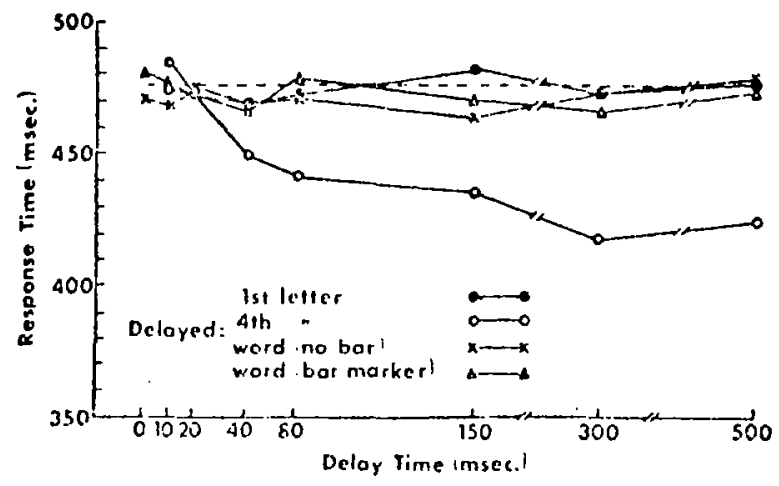

Fig. 2. Mean response time as a function of delay condition and delay interval. (Delay time subtracted from response time.)

delayed by one of seven intervals. To control for the possibility that the act of pressing the switch might also serve as a priming stimulus, a second condition presented the whole word without the marker either simultaneously with the switch press or delayed. Conditions delaying the first and fourth letter were also employed as a partial replication of Experiment I.

\section{EXPERIMENT II}

\section{Method}

\section{Subjects}

Ss were four college-age students (two female), all of whom had normal or corrected to normal vision. One had participated in the previous experiment. The other three had no previous experience in visual experiments. All were paid to participate.

\section{Apparatus and Stimuli}

Apparatus was the same as in the previous experiment. Stimuli were prepared using the same materials as before.

Stimuli were again constructed from 10 four-letter base words, 7 of which were the same as in the previous experiment. The base words SLOW, FARE, and LURE were replaced with CORE, BALE, and PINS. Their variants are, with the first letter changed, BORE, TALE, and TINS; changing the fourth letter gives CORD, BALD, and PINK. For the seven repeated words, the first and fourth letter variants used were also the same as previously used. Of the 30 words formed by the 10 base words and their 2 variants used in this experiment, 20 were chosen for use in the conditions in which the entire word was delayed. These words were:

$\begin{array}{llll}\text { LANE } & \text { PINS } & \text { FAME } & \text { BINS } \\ \text { RICE } & \text { NICE } & \text { BORE } & \text { LAMB } \\ \text { BEAT } & \text { YARD } & \text { TALE } & \text { MATS } \\ \text { MATE } & \text { FIND } & \text { LAND } & \text { BALD } \\ \text { CORE } & \text { HEAT } & \text { HARP } & \text { PINK }\end{array}$

For the condition in which a bar marker appeared immediately, followed by the entire word, a black bar, $.5 \mathrm{deg}$ of angle long and .1 deg of angle in width, appeared centered $1 / 2 \mathrm{deg}$ below the word. All other physical aspects of the stimulus presentation were the same as in the previous experiment.

\section{Procedure}

For the two conditions in which the entire word was presented, either with or without the bar marker, the word either appeared immediately (zero delay) or delayed by 10,20 , $40,80,150,300$, or 500 msec. For the letter-delay conditions, the zero delay was not used, as the same effect was obtained in the two word-delay conditions. Other delay times were the same.

Each experimental session consisted of 12 blocks of 10 trials each. Within each block, all trials used the same conditions and delay time. There were 10 experimental sessions in all, with half of each session consisting of word delays and half of letter delays, with the zero delay considered as a member of either group. Presentation of the conditions and two different letter delays was counterbalanced across sessions by a modified Latin square design, with each $S$ entering the sequence at a different place. Ten sessions yielded four blocks of trials for each $S$ for each possible combination of word and letter conditions with delay times.

Ss were again given two practice sessions before beginning the experiment proper. All other aspects of the presentation procedure were the same as in Experiment $l$.

\section{Results and Discussion}

Figure 2 shows the response times for the four conditions averaged across Ss. These are shown from the time of onset of the delayed stimulus. Delay of the fourth letter again appears to offer an advantage to the Ss, while delaying the entire word leads to RTs almost identical with those obtained for delay of the first letter. A three-way ANOVA using Ss by first letter delay plus the two word-delay conditions by delay interval (simultaneous presentations omitted) showed that these three conditions were not significantly different. Inclusion of the fourth letter delay did yield a significant effect of conditions $[F(3,9)=5.95, p<.02]$ as well as a significant Conditions by Delay Interval interaction $[F(18,54)=3.61, p<.001$. As in the previous experiment, the other main effects were also significant. There was also indication of an Ss by Conditions interaction. Examination of the individual $S$ data showed this to be due to the $S$ used in the previous experiment who was able to respond somewhat faster when the first letter was delayed than when the entire word was delayed, while the other three Ss took longer for this condition. This may have been due to the additional practice or to the fact that he was an exceptionally fast-responding $\mathrm{S}$. He remarked that at the two longer delay times he had already narrowed his response to the two alternatives while waiting for the first letter, which may have given him an advantage over those Ss not employing this strategy.

Examination of Fig. 2 shows that, for the three similar conditions, RTs are almost an exact addition of the average RT for the undelayed presentation of the entire word (approximately $475 \mathrm{msec}$ ) and for the delay interval. Knowing the last three letters of the word does not appear very helpful, even though the number of 
alternatives is greatly reduced by this information. Delay of the fourth letter, on the other hand, again appears to speed up the process. For example, at a delay of $80 \mathrm{msec}$, the average RT is only $520 \mathrm{msec}$ rather than $555 \mathrm{msec}$. This gain is comparable to that shown in the first experiment when any letter other than the first is delayed. As long as the first letter is present, the $\mathbf{S}$ seems to be able to "go to work" immediately on processing the word.

Two possible advantages accrue from the presence of the first letter. In the unlikely event that words are stored in the "internal lexicon" in alphabetical order, it would be helpful merely to know what the first letter is in order to retrieve the proper word from the memory store. If, on the other hand, a kind of fast implicit speech is involved in reading a word, the sound of the first letter may be the important variable. In all the words used in the first two experiments, the sound of the first letter was never dependent on knowledge of the other letters.

As a preliminary check of the effect of a sequential pronunciation dependency within words, an analysis was run on the RTs for the third letter delayed condition using two words from the first experiment in which the pronunciation of the second letter depended on knowledge of the third letter (FARE-FADE, MATE-MARE) and two where it was not dependent (LANE-LAKE, RICE-RIDE). RTs were faster at all delay intervals for the nondependent case, and the results were marginally significant $[F(1,3)=8.90, p<.06$. These results must be interpreted with caution, since the possibility exists that the two nonindependent words might simply have had a quicker initial vocalization due to the phonemes involved.

If it is the sound of the first letter which is important, then delaying a second letter which determines the sound of the first should produce RTs similar to those found when the first letter itself is delayed. In order to be comparable to the previous experiments, it was necessary that the second letter be a vowel, thus eliminating the use of initial consonant blends or digraphs to achieve the desired dependency. There are, unfortunately, only two initial letters in English orthography, $\mathrm{C}$ and $\mathrm{G}$, which demonstrate this dependency and still maintain their integrity as individual letters. The use of words beginning with $\mathrm{S}$ which becomes SH in such words as SURE was discarded as questionable both in terms of the before-mentioned constraints and because of the ease with which an $S$ could slide from one version to the other in midresponse. With some difficulty, seven appropriate four-letter base words were found. Conditions presented in the third experiment included delay of the second letter, upon which the first letter sound depended, delay of the first and third letters, and delay of the whole word.
Table 2

Base Words and Variants Used in Experiment III

\begin{tabular}{llcc}
\hline \multirow{2}{*}{$\begin{array}{c}\text { Base } \\
\text { Words }\end{array}$} & \multicolumn{3}{c}{ Letter Delayed } \\
\cline { 2 - 4 } GUMS & HUMS & GEMS & GUTS \\
GONE & LONE & GENE & GORE \\
CELL & BELL & CALLL & CEIL \\
CANT & PANT & CENT & CART \\
CODE & MODE & CẼDE & CORE \\
CUTE & LUTE & CITE & CURE \\
COLT & BOLT & CELLT & CoST \\
\hline
\end{tabular}

\section{EXPERIMENT III}

\section{Method}

\section{Subjects}

Ss were four college-age students, three of whom had participated in one or the other of the previous experiments. The fourth had not previously taken part in any visual experiment. The Ss were paid for their work.

\section{Apparatus and Stimuli}

Apparatus was again the same and stimuli were prepared as in the previous experiments.

Within the constraint that changing the second letter changes the pronunciation of the first letter, seven base words were found for which the first, second, and third letters could be changed and produce an alternate word. Changes of the fourth letter to produce reasonable alternate words were difficult or impossible with this base-word group. The seven base words and their three sets of variants appear in Table 2.

\section{Procedure}

Four delay conditions were used in this experiment: delay of the first, second, or third letter and delay of the entire word. Delay times used for each condition were $0,20,40,80,150$, and $300 \mathrm{msec}$.

Each experimental session consisted of eight blocks of 14 trials each, two within each delay condition. Each session used all eight delay times. Order of presentation of delay by condition combinations was counterbalanced across sessions by a modified Latin square design. Six experimental sessions yielded two blocks of trials for each $S$ for each combination of delay time by delay condition. Since only 14 words were involved in each variant set and it was thought that Ss might be able to recall which words had been presented during a block, presentation was randomized across sessions within a delay time by delay condition combination. All 28 words were used to form the set of words for the whole-word delay condition. At the conclusion of the six regular experimental sessions, an additional session was run using only the seven base whole words at each delay time in blocks of 14. This was done for purposes of comparison with performance on those words alone under the other conditions.

\section{Results and Discussion}

This experiment was by far the most difficult from the point of view of the Ss. Delay of the second letter, 


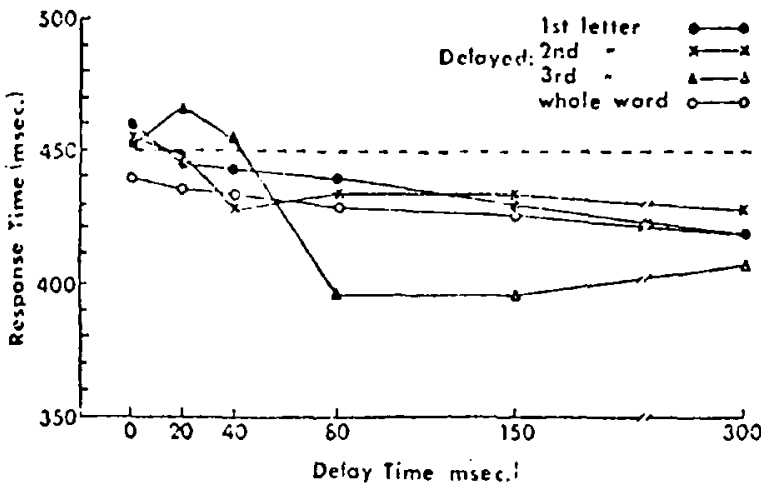

Fig. 3. Mean response time as a function of delay condition and delay interval. (Delay time subtracted from response time.)

and with it the knowledge of how the first letter should sound, seemed to be very distressing. As Fig. 3 illustrates. by the 80 -msec delay time, performance with the second letter delayed was poorer than when the entire word was delayed and continued to be so at the 150 - and $300-\mathrm{msec}$ delays. For the purposes of this comparison, response times for the base words only in all conditions were used. This approach was adopted. since some of the rather odd words used as variants caused some of the Ss difficulty. For the 80-, 150-, and 300 -msec delay intervals, delay of the third letter again reduces response times. The rather strange hump in the third-letter curve, preceding the $80 \cdot \mathrm{msec}$ delay, was attributable almost entirely to one $S$, and there is no apparent explanation for his difficulty at these delay times. This $\mathrm{S}$ also experienced considerably more difficulty when the first letter was delayed, relative to his whole-word performance, than any other $S$. This does, of course, give a large Ss by Conditions interaction effect when an ANOVA is done for all conditions by delay times $[F(9.45)=4.58, p<.001]$. An abbreviated three-way ANOVA was therefore done, using only the second- and third-letter delay conditions and the three longest delay times for the four Ss. Results showed conditions to be significant $[F(1,3)=13.89, p<.04]$, with the other main effects also significant. None of the interactions approached significance.

\section{DISCUSSION}

Results of all three experiments clearly confirm the importance of the first letter to recognition ${ }^{1}$ of a word. Somewhat surprising is the fact that presentation of the last three letters offers little help compared to delay of all of the letters, even though this reduces the alternatives to only two in the case of these studies. The most interesting finding is that knowledge of the sound of the first letter, or of the sound relationships among the first letters, facilitates response times relative to response times when the entire word was delayed. Clearly, the Ss were able to begin processing of the word on the basis of these cues. It was not necessary to wait for the entire word to be present and identified. The amount of facilitation in response time accrueing from knowledge of the sound of the first letter was comparable across all three experiments at approximately $30 \mathrm{msec}$. Table 3 presents these comparisons for the $80-\mathrm{msec}$ delay interval for all experiments. This interval was chosen since, by the 80-msec delay time, the fact that a letter is missing at first is clearly discernible to all Ss. This permits any advantages accruing from the delay to be apparent as well as any disadvantages which might occur due to a disruptive effect of the delay. Those times marked with an asterisk $\left(^{*}\right)$ represent the cases in which the first letter sound information was available. Also shown are the comparisons for the case in which the third letter did or did not determine the pronunciation of the second letter. It appears that when the third letter is not crucial to the sound of the second, the $S$ is able to progress beyond the first letter with his processing. This reduces his response time after the appearance of the third letter by almost another $30 \mathrm{msec}$. Additional facilitation does not appear in the other response time averages for third and fourth letter delays, since these data include both letters which determine previous letter sounds (except for the first letter) and let ters which do not.

These findings suggest that the Ss are using their knowledge of the sound-spelling correspondences in English to progress sequentially from left to right in the most efficient way possible. About $30 \mathrm{msec}$ per sound gives the $\mathrm{S}$ time to make some kind of implicit response and prepare for the overt response. The implicit response itself is probably somewhat faster. Eriksen, Pollack, and Montague (1970) studied the response times to one-, two-, and three-syllable words. They found that the second and third syllables added about 11 msec each to the response times. The difference between these figures and those obtained in the present study may be due to the difference in task, or it may reflect to some degree the lesser amount of information per sound for longer words reported by Black (1954). Although it has been suggested that implicit speech takes as long as overt speech (Landauer. 1962), this seems relevant only to a kind of silent reading conducted as if one were reading aloud. Even the most skilled readers, whose rate far exceeds that of oral reading. still report that there is a kind of auditory analog present. The exact nature of this

Table 3

Comparative Response Times at $80 \mathrm{Msec}$ Delay

\begin{tabular}{llll}
\hline & \multicolumn{3}{c}{ Experiment } \\
\cline { 2 - 4 } & I & II & III \\
\hline Word delayed & & 550 & 510 \\
First letter delayed & 534 & 555 & 520 \\
Second letter delayed & $495^{*}$ & & 514 \\
Third letter delayed & $495^{*}$ & & $476^{*}$ \\
Fourth letter delayed & $498^{*}$ & $520^{*}$ & \\
Third determines second & 495 & & \\
Third does not determine second & $469^{*}$ & & \\
\hline
\end{tabular}


kind of implicit speech is still not clear. but it appears to be an essential part of the reading process for most people.

It might be argued that a fast verbal response to a single word is very different from reading a page of text to oneself, and indeed it is. It would be difficult, however. to conceive of this task as being done in a manner entirely different from normal reading. In the early stages of learning to read, a child reads many single words or attacks a sentence a word at a time. Many students of the processes involved in learning to read have found that, for the normal child, learning the sound-spelling correspondences seems the most fruitful approach (e.g.. Chall. 1970; Weir \& Venezky, 1968; Ruddell, 1968). For the child who cannot learn to read (dyslexic), the chief difficulty appears to be an inability to learn to recognize sequential relationships of temporally or spatially ordered stimuli and to transpose from one to the other (Isom, 1969). Isom states, "This ability to perform 'automatic' written symbol-spoken sound correspondence must' precede effective reading for the acquisition of meaning [p. 5]." The present study lends support to this view, as the basic ability that skilled readers bring to word recognition seems to be an almost instantaneous decoding of the sound-spelling patterns of their language.

\section{REFERENCES}

Aderman. D.. \& Smith, E. E. Expectancy as a determinant of functional units in perceptual recognition. Cognitive Psychology, 1971, 2, 117-129.

Bishop, C. H. Transfer effects of words and letter training in reading. Journal of Verbal Learning \& Verbal Behavior, 1964, 3, 215-221.

Black, J. W. The information of sounds and phonetic digrams of one- and two-syllable words. Journal of Speech \& Hearing Disorders, 1954, 19, 397-411.

Broerse, A. C., \& Zwaan, E. J. The information value of initial letters in the identification of words. Journal of Verbal Learning \& Verbal Behavior, 1966, 5, 441446.

Bruner, J. S., \& O'Dowd, D. A note on the informativeness of parts of words. Language \& Speech, 1958, 1, 98-101.

Chall, J. S. Learning and not learning to read: Current issues and trends. In F. A. Young and D. B. Lindsley (Eds.), Early experience and visual information processing in perceptual and reading disorders. Washington, D.C: National Academy of Sciences, 1970.

Eriksen, C. W., Pollack, M. D., \& Montague, W. E. Implicit speech: Mechanism in perceptual encoding? Journal of Experimental Psychology, 1970, 84, 502-507.

Gibson, E. J. Principles of perceptual learning and development. New York: Appleton-Century-Crofts, 1967.

Gibson, E. J., Osser, H., \& Pick, A. D. A study in the development of grapheme-phoneme correspondences. Journal of Verbal Learning \& Verbal Behavior, 1963, 2, 142-146.
Gibson, E. J., Pick, A., Osser, H., \& Hammond, M. The role of grapheme-phoneme correspondence in the perception of words. American Journal of Psychology, 1962, 75, 554-570.

Gibson, E. J., Shurcliff, A., \& Yonas, A. The role of pronounce-ability in perception of pseudo-words by hearing and deaf subjects. Project Literacy Reports, 1966, No.7, 62-72.

Huey, E. B. The psychology and pedagogy of reading. New York: MacMillan, 1908. Reprinted-Cambridge, Mass:M.I.T. Press, 1968.

Isom, J. B. An interpretation of dyslexia-a medical viewpoint. In G. D. Spache (Ed.), Reading disability and perception. Proceedings of the Thirteenth Annual Convention, International Reading Association, Newark, Delaware, 1969.

Jones, M. H. Some thoughts on perceptual units in language processing. In K. S. Goodman (Ed.), The psy'cholinguistic nature of the reading process. Detroit: Wayne State University Press, 1965.

Kolers, P. A., \& Katzman, M. T. Naming sequentially presented let ters and words. Language \& Speech, 1966, 9, 84-95.

Landauer, T. K. Rate of implicit speech. Perceptual \& Motor Skills, 1962, 15, 646 .

Melville, J. R. Word-length as a factor in differential recognition. American Journal of Psychology, 1957, 70, 316-318.

Oléron, P. Sur les stratégies dans l'identification des mots. Journal de Psychologie Normale et Pathologique, 1970, 3, 313-323.

Reicher, G. M. Perceptual recognition as a function of meaningfulness of stimulus material. Journal of Experimental Psychology, 1969, 81, 275-280.

Ruddell, $R$. The relation of regularity of grapheme-phoneme correspondences and language structure to achievement in first grade reading. In $\mathrm{K}$. S. Goodman (Ed.), The psycholinguistic nature of the reading process. Detroit: Wayne State University Press, 1965.

Sumby, W. H., \& Pollack, Y. Short-time processing of information. HFORL Report TR-54-6. Washington, D.C: U.S. Government Printing Office, 1954.

Weir, R. H., \& Venezky, R. L. Spelling-to-sound patterns. In K. $\mathrm{S}$. Goodman (Ed.), The psycholinguistic nature of the reading process. Detroit: Wayne State University Press, 1968.

Wheeler, D. D. Processes in word recognition. Cognitive Psychology, 1970, 1, 59-85.

\section{NOTE}

1. It is obviously true that what has been measured in these experiments is the time to begin an overt response. "Recognition" in these circumstances is confined to whatever internal processes must be carried out in order for an $S$ to be able to make the appropriate response. Ss apparently must be able to recognize the proper sound relationships between the letters in order to begin their overt responses. As most students are aware, one can "recognize" all the words in a passage in this limited way and the meanings escape him completely. The reverse does not appear to be possible (cf. Isom, 1969).

(Received for publication February 7, 1973; revision received July $27,1973$. ) 